\title{
Post-Allogeneic Bone Marrow Transplant
}

National Cancer Institute

\section{Source}

National Cancer Institute. Post-Allogeneic Bone Marrow Transplant. NCI Thesaurus.

Code C125200.

The time subsequent to an allogeneic bone marrow transplant. 